\title{
Tea consumption reduces the incidence of gallbladder cancer based on a meta-analysis of epidemiologic studies
}

\author{
ZHANG YaQin ${ }^{1 \dagger}$, ZHANG Yi ${ }^{1{ }^{1 \dagger *}}$, WU XinJuan ${ }^{2}$, XIANG Min $^{2} \&$ MA YuFen ${ }^{2 *}$ \\ ${ }^{1}$ Department of General Surgery, Peking Union Medical College Hospital, Chinese Academy of Medical Sciences \& Peking Union Medical \\ College, Beijing 100730, China; \\ ${ }^{2}$ Nursing department, Peking Union Medical College Hospital, Chinese Academy of Medical Sciences \& Peking Union Medical College, \\ Beijing 100730, China
}

Received March 16, 2015; accepted April 4, 2015; published online August 21, 2015

Citation: Zhang YQ, Zhang Y, Wu XJ, Xiang M, Ma YF. Tea consumption reduces the incidence of gallbladder cancer based on a meta-analysis of epidemiologic studies. Sci China Life Sci, 2015, 58: 922-924, doi: 10.1007/s11427-015-4896-2

\section{Dear Editors,}

Gallbladder cancer comprises mostly of biliary tract malignancy which is the seventh most common digestive system cancers. The prognosis of gallbladder cancer is poor, whose five-year survival was reported 5\%-10\%. Many risk factors for gallbladder cancer have been identified in plenty of studies. There has been a definitive association between gallstones and gallbladder cancer. Gallbladder polyps and bacterial-induced chronic inflammation are also established risk factors for gallbladder cancer. Some specific occupational exposures, petroleum refining for example, increase the incidence of gallbladder cancer. Other associated risk factors include sex, age, postmenopausal status, cigarette smoking and so on, most of which might cause the mucosal lesion of gallbladder. Tea, which derived from the leaves of the plant Camellia sinensis, is one of the most popular beverages worldly. The green tea and black tea are the most popular. Numerous in vitro and animal studies have proved that the compounds in tea, especially polyphenols, play a significant antimutagenic role in various cancer. Up to date, there have been several epidemiologic studies on the association between tea and gallbladder cancer, which are not consistent or even contradictory. There has been no system-

$\dagger$ Contributed equally to this study

*Corresponding author (email: zhangyi4852@sina.com; yumafen@126.com) atic meta-analysis to elucidate the role of tea consumption on gallbladder cancer so far. Therefore, we carry out this meta-analysis to evaluate the association.

We concluded an electronic literature search on tea and gallbladder cancer in PubMed, EMBASE and Medline to identify relevant human adult studies without language limitation until December 2014. The used terms were ("tea" and ("gallbladder cancer" or "gallbladder carcinoma" or "gallbladder tumor")). Additionally, we reviewed the references from retrieved articles for potential studies. The epidemiological studies which addressed the association between tea intake and gallbladder cancer were selected as potential subjects. If the study provides no original data or insufficient information on the odds ratio $(O R)$ or relative risk $(R R)$, and their corresponding $95 \%$ confidence intervals $(C I s)$, we excluded it. Two independent reviewers read the abstracts or full-text articles to assess the eligibility of studies in a standardized manner. We extracted important information including study design, country of origin, years of publication, origin of control, number of cases and control, sex distribution, kinds of tea, types of cancer, comparison of exposure level, and potential confounding variables adjusted. The estimates of odds ratio $(O R) /$ relative risk $(R R)$, their associated $95 \% C I s$, and $P$-values were also extracted by us. According to the equation: $S E=\left(\ln O R / R R \_\right.$upper $-\ln O R / R R$ lowel)/3.92, we calculated the $S E$ of the $\ln O R / R R$ extracted from studies (Table S1). We calculated the overall $O R$ and 
their 95\% CI among different subgroups of three studies by using fixed effect model. We evaluate heterogeneity with $I^{2}$ and $0 \%$ means that there is no observed heterogeneity. We used the random effect model to calculate the summary $O R$ and its $95 \%$ CI with suspecting heterogeneity. When we found that the heterogeneity was not obvious, we calculated the summary $O R$ and its $95 \%$ CI using fixed effect model. We evaluated the impact of the changes on the pooled $O R s$ by study design as prior hypotheses to explain heterogeneity through subgroup analyses. We considered the funnel plot to be asymmetrical if the intercept of the regression line deviated from zero with $P<0.10$ and the publication existed. We used the Cochrane Collaboration software RevMan5.0 to analyze the extracted data with fixed or random effects model analysis . STATA (StataCorp, College Station, USA) was used to conduct the Egger regression asymmetry test by using the metabias command.

The electronic search yielded 246 references, of which 14 were included after abstract review. Finally, five studies were included in the meta-analysis, including two cohort studies $[1,3]$ and three case-control studies (Table S2) [2,4,5]. All literatures were published in English. All studies adjusted for age. Studies differed in adjustment for other confounding variables like sex, education level and so on (Table S2). The adjusted $O R / R R$ for the ever category of tea intake varied considerably, which ranged from 0.41 to 1.4 . Two studies provided $P$ value [3,4], one of which reached the usual threshold of $P=0.05$ in the association between tea intake and gallbladder cancer [4]. No significant heterogeneity among the study results $\left(I^{2}=44 \% ; P=0.13\right)$ was observed. Overall summary $O R$ using the fixed-effect model showed a $23 \%$ reduction in risk of gallbladder cancer associated with tea intake (summary $O R=0.77 ; 95 \%$ $C I=0.63-0.94 ; P=0.009$ ) (Figure S1). No publication bias was observed among eligible studies. Visualization of funnel plot was symmetrical (Figure 1).

The overall summary $O R$ in our study exhibits that tea consumption can decrease risk of gallbladder cancer (summary $O R=0.77 ; 95 \% C I=0.63-0.94$ ). The protective effect still exists in subgroup analysis of case-control studies (OR=0.69; 95\% CI=0.54-0.89), but prospective cohort studies are insufficient to support the relationship. The inverse associations between tea drinking and cancer risks have been reported in several previous quantitative meta-analysis. Nevertheless, the null associations have also persisted. As we have mentioned above, tea contains lots of antitumor components. The major components in green tea are polyphenols, which play a critical role in cancer chemoprevention, include four catechins, namely (-)-epigallocatechin-3-gallate (EGCG), (-)-epigallocatechin (EGC), (-)-epicatechin-3-gallate (ECG) and (-)-epicatechin (EC). EGCG is the most active and abundant catechins. Numerous studies have indicated that EGCG participates in multiple signal pathways related to cell cycle, cell apoptosis and tumorigenesis. EGCG inhibits the activation of epidermal

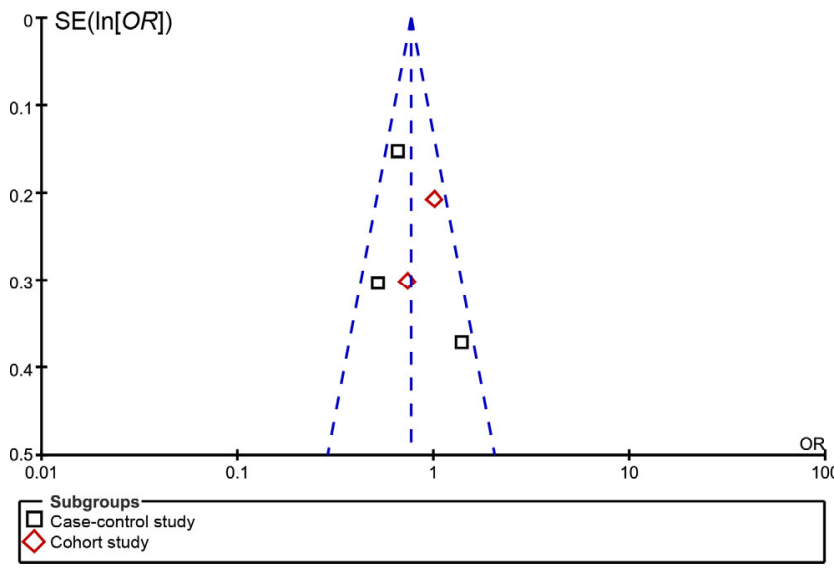

Figure 1 Funnel plot of studies evaluating the association between tea intake and gallbladder cancer risks. Dot lines are $95 \%$ pseudo-confidence intervals. SE, standard error; OR, odds ratio.

growth factor receptor (EGFR), insulin-like growth factor 1 receptor (IGF-1R) and vascular endothelial growth factor receptor (VEGFR1), which are all members of receptor tyrosine kinases, then blocks cell proliferation and induces apoptosis in various cancer cells. Additionally, the major constituents of black tea are oligomeric polyphenols (theaflavins) and polymeric polyphenols (thearubigins), which have antioxidant function and potential antitumor effect. However, there are few researches on the detailed mechanism of catechins to gallbladder cancer in-vitro or animal experiment. There are also several limitations we should take into consideration. Firstly, all date are from observational studies with different study populations, study design and various definition of tea intake. Moreover, the number of the included studies and the heterogeneity between them weaken the confirmation of our results. Additionally, most dietary studies measure the recent estimates of dietary intake, whereas the development of cancer takes several decades. We have just included three case-control studies and two cohort studies, which may not have the sufficient power. Case-control studies may lower summary $O R$ because of recall and selection biases. However prospective studies can avoid such weaknesses. Therefore the accurate ORs might be higher than that gained in these studies.

In summary, we conclude that tea could reduce the incidence of gallbladder cancer by $23 \%$ based on the meta-analysis of several epidemiological studies. Due to the inconsistency in results between case-control studies and cohort studies, larger prospective studies with rigorous design should be put forward to further study.

The authors declare that they have no conflict of interest.

1 Nechuta S, Shu XO, Li HL, Yang G, Xiang YB, Cai H, Chow WH, Gao YT, Zheng W. Prospective cohort study of tea consumption and risk of digestive system cancers: results from the Shanghai women's health study. Am J Clin Nutr, 2012, 96: 1056-1063

2 Zhang XH, Andreotti G, Gao YT, Deng J, Liu E, Rashid, Wu K, Sun 
L, Sakoda LC, Cheng JR, Shen MC, Wang BS, Han TQ, Zhang BH, Gridley G, Fraumeni JF Jr, Hsing AW. Tea drinking and the risk of biliary tract cancers and biliary stones: a population-based case-control study in Shanghai, China. Int J Cancer, 2006, 118: 3089-3094

3 Nagano J, Kono S, Preston DL, Mabuchi K. A prospective study of green tea consumption and cancer incidence, Hiroshima And
Nagasaki (Japan). Cancer Causes Control, 2001, 12: 501-508

4 Zatonski WA, La Vecchia C, Przewozniak K, Maisonneuve P, Lowenfels AB, Boyle P. Risk factors for gallbladder cancer: a Polish case-control study. Int J Cancer, 1992, 51: 707-711

5 La Vecchia C, Negri E, Franceschi S, D’Avanzo B, Boyle P. Tea consumption and cancer risk. Nutr Cancer, 1992, 17: 27-31

Open Access This article is distributed under the terms of the Creative Commons Attribution License which permits any use, distribution, and reproduction in any medium, provided the original author(s) and source are credited.

\section{Supporting Information}

Table S1 Logarithmic $O R$ or $R R(\ln (O R / R R))$ and its $S E$ for the meta-analysis

Table S2 Summary of studies included in the meta-analysis

Figure S1 Summary estimates of the association between tea intake and gallbladder cancer risks sorted by effect estimate.

The supporting information is available online at life.scichina.com and link.springer.com. The supporting materials are published as submitted, without typesetting or editing. The responsibility for scientific accuracy and content remains entirely with the authors. 